\title{
Contributi allo studio dell' esistenza di soluzioni periodiche per i sistemi di equazioni differenziali ordinarie $\left(^{*}\right)$.
}

\author{
Memotia di Gaetano Villari (a Firenze)
}

Sommario. - Si considera il problema dell'esistenza di soluzioni periodiche per $i$ sistemi, non necessariamente periodici, del tipo

$$
\dot{x}=A(t) x+f(t, x),
$$

e lo si riconduce allo studio dell'esistenza di soluzioni comuni a due equazioni funzion ali. Nel caso della periodicità di $A(t)$ e $f(t, x)$, ed in particolare in quello delle equazioni del terzo ordine del tipo di Aizerman, si ottengono criteri di esistenza di soluzioni armoniche.

1. - La presente ricerca è dedicata al problema dell'esistenza di soluzioni periodiche per un sistema di equazioni differenziali ordinarie della forma

$$
\dot{x}=A(t) x+f(t, x)
$$

ove $x=x(t)$ rappresenta un vettore le oui componenti $x_{i}(t) \quad(i=1,2, \ldots, n)$ sono funzioni della variabile reale $t ; \dot{x}=d x / d t ; A(t)=\left(\alpha_{i j}(t)\right)$ ̀े una matrice $n \times n$ reale, definita e continua nell' intervallo $R: t>T \geq-\infty ; f(t, x)$ ̀े un vettore $n \times 1$ definito e continuo nella regione $S: R \times E^{n}$ dello spazio euclideo a $n+1$ dimensioni delle variabili $\left(t, x_{1}, \ldots, x_{n}\right)$.

Sia $\omega>0$ il periodo delle eventuali soluzioni periodiche di (1) di cui si vuole studiare l'esistenza.

Non faremo in un primo tempo alcuna particolare ipotesi di periodicità, con periodo $\omega$, per la matrice $A(t)$ e per il vettore $f(t, x)$.

Sarà invece essenziale, nella presente trattazione, una ipotesi concernente la natura delle soluzioni del sistema omogeneo

$$
\dot{y}=A(t) y
$$

associato al sistema (1), e che troverà giustificazione in quanto sarà detto nel successivo n. 3 .

(\%) Ricerua eseguita nell'ambito del Gruppo di Ricerca n. 11 del C.N.R. 
Precisamente, fissato $t_{0} \in R$, ed indicando con $Y(t)$ la matrice fondamentale di (2) tale che $Y\left(t_{0}\right)=I$ (I matrice $n \times n$ unità), supporremo che risulti soddisfatta la condizione

$$
\operatorname{det}[Y(t)-Y(t+\omega)] \neq 0, \quad \text { per } t>T
$$

Tale condizione, qualora si suppongano la matrice $A(t)$ e il vettore $f(t, x)$ periodici rispetto a $t$ con periodo $\omega$, si riduce all'ordinaria ipotesi di non risonanza; e si vede facilmente che essa equivale ad affermare la non esistenza di soluzioni $y(t)$ non nulle del sistema (2) per le quali si ha

$$
y(t+\omega)=y(t), \quad \text { per qualche } t \in(T, \infty)
$$

2. - Ciò posto, sia $x=u(t)$ una soluzione del sistema (1) periodica di periodo $\omega$; com'è noto (cfr. [8], p. 470) $\left({ }^{1}\right)$, essa verifica identicamente l'uguaglianza

$$
u(t)=Y(t) u^{0}+Y(t) \int_{t_{0}}^{t} Y^{-1}(\tau) f[\tau, u(\tau)] d \tau,
$$

ove $u^{0}$ rappresenta il valore assunto da $u(t)$ per $t=t_{0}$.

Dalla (3) e dalla (4) si ha subito che il vettore

$$
\begin{gathered}
F(t)=[Y(t)-Y(t+\omega)]^{-1} Y(t+\omega) \int_{t}^{t+\omega} Y^{1}(\tau) f[\tau, u(\tau)] d \tau- \\
-\int_{t_{0}}^{t} Y^{-1}(\tau) f[\tau, u(\tau)] d \tau=u_{0}
\end{gathered}
$$

è costante, e poichè, derivando la (5), con semplici calcoli si ha $\left({ }^{2}\right)$

$$
\begin{gathered}
\dot{F}(t)=[A(t)-A(t+\omega)] Y(t)[Y(t)-Y(t+\omega)]^{-1} Y(t+\omega) \\
\int_{t}^{t+\omega} Y^{-1}(\tau) f[\tau, u(\tau)] d \tau+f[t, u(t)]-f[t+\omega, u(t+\omega)]
\end{gathered}
$$

(1) I numeri in parentesi quadra si riferiscono all'elenco bibliografico posto in fondo al lavoro.

(2). Basta osservare che $\dot{Y}(t)=A(t) Y(t)$ e servirsi di note proprietà delle matrici. 
ne segue per $u(t)$ la condizione

$$
[A(t)-A(t+\omega)] Y(t)[Y(t)-Y(t+\omega)]^{-1} Y(t+\omega) \int_{t}^{t+\omega} Y^{-1}(\tau) f[\tau, u(\tau)] d \tau+
$$

$$
+f[t, u(t)]-f[t+\omega, u(t+\omega)]=0 \text {. }
$$

D'altra parte, sostituendo nella (4) il valore di $u_{0}$ dato dalla (5), si ha pure

$$
u(t)=Y(t)[Y(t)-Y(t+\omega)]^{-1} Y(t+\omega) \int_{i}^{t+\omega} Y^{-1}(\tau) f[\tau, u(\tau)] d \tau .
$$

La (7) e la (8) sono quindi necessarie perchè il vettore $u(t)$ sia soluzione periodica di periodo $\omega$ del sistema (1).

Si vede facilmente che tali condizioni sono altresì sufficienti.

Infatti, la (8), tenendo conto della (5), può scriversi

$$
u(t)=Y(t) F(t)+Y(t) \int_{t_{0}}^{t} Y^{-1}(\tau) f[\tau, u(\tau)] d \tau
$$

e derivando rispetto a $t$, dalla (6) e dalla (7) si ottiene

$$
\dot{u}(t)=A(t) u(t)+f[t, u(t)] .
$$

Inoltre, sempre dalla $(9)$, si ha

$$
u(t+\omega)=Y(t+\omega) F(t)+Y(t+\omega) \int_{t_{0}}^{t+\omega} Y^{-1}(\tau) f[\tau, u(\tau)] d \tau
$$

e per la (5) e la (8):

$$
\begin{gathered}
u(t+\omega)=Y(t+\omega)[Y(t)-Y(t+\omega)]^{-1} Y(t+\omega) \int_{t}^{t+\omega} Y^{-1}(\tau) f[\tau, u(\tau)] d \tau+ \\
+Y(t+\omega) \int_{t}^{t+\omega} Y^{-1}(\tau) f[\tau, u(\tau)] d \tau= \\
=Y(t)[Y(t)-Y(t+\omega)]^{-1} Y(t+\omega) \int_{t}^{t+\omega} Y^{-1}(\tau) f[\tau, u(\tau)] d \tau=u(t) .
\end{gathered}
$$

Concludendo, si ottiene il

Teorena 1. - Se per il sistema (2) è valida la condizione (3), l'insieme 
delle soluzioni periodiche di periodo o del sistema (1) coincide con quello dei vettori $u(t)$ che soddisfano la (7) e la (8).

Si osservi infine che, limitandosi com'è naturale allo spazio $\Omega$ dei vettori periodici di periodo $\omega$, tale Teorema può assumere le seguente forma equivalente, maggiormente adatta per le applicazioni che ne saranno fatte:

Teorema 1'. - Se per il sistema (2) è soddisfatta la (3), il sistema (1) presenta soluzioni periodiche di periodo $\omega$ se, e soltanto se, non è vuoto in $\mathbf{\Omega}$ $l$ 'insieme dei veltori $x(t)$ per cui :

$$
\begin{gathered}
x(t)=Y(t)[Y(t)-Y(t+\omega)]^{-1} Y(t+\omega) \int_{t}^{t+\omega} Y^{-1}(\tau) f[\tau, x(\tau)] d \tau \\
{[A(t)-A(t+\omega)] x(t)+f[t, x(t)]-f[t+\omega, x(t)]=0\left(^{3}\right) .}
\end{gathered}
$$

3. - 亡̀ opportuno osservare che, qualora non risulti soddisfatta la condizione (3), si perde in generale la possibilità di impostare il problema della esistenza di soluzioni periodiche del sistema (1) in modo analogo a quanto è stato fatto coi Teoremi del numero precedente.

In effetti si vede facilmente che un vettore $x(t)$, soluzione periodica di periodo $\omega$ del sistema (1), oltre a soddisfare la (11), dovrà verificare per $t>T$ la condizione

$$
[Y(t)-Y(t+\omega)] Y^{-1}(t) x(t)=Y(t+\omega) \int_{t}^{t+\omega} Y^{-1}(\tau) f[\tau, x(\tau)] d \tau,
$$

o la sua equivalente

$$
x(t)=Y(t+\omega) Y^{-1}(t) x(t)+Y(t+\omega) \int_{t}^{t+\omega} Y^{-1}(\tau) f[\tau, x(\tau)] d \tau
$$

D'altra parte, derivando la (12) rispetto a $t$, e supponendo che il vettore $x(t)$ sia periodico di periodo $\omega$ e tale da soddisfare la (11), si ottiene

$$
[Y(t)-Y(t+\omega)] Y^{-1}(t)\{\dot{x}(t)-A(t) x(t)-f[t, x(t)]\}=0
$$

il che non implica necessariamente che $x(t)$ sia soluzione del sistema (1).

( $\left.{ }^{3}\right)$ Tale condizione puó d'altra parte ottenersi direttamente dalla (1) e dalla supposta periodicità di $x(t)$ e $\dot{x}(t)$. 
In realtà, se il vettore $x(t) \in \Omega$ verifica la $\left(12^{\prime}\right)$, può solo affermarsi che anche il vettore

$$
G(t)=Y^{-1}(t) x(t)-\int^{t} Y^{-1}(\tau) f[\tau, x(\tau)] d \tau
$$

è periodico di periodo w, ma non necessariamente costante, e dalla (13) si ottiene

$$
\dot{x}(t)=A(t) x(t)+f[t, x(t)]+Y(t) \dot{G}(t)
$$

che coincide con la (1) se, e soltanto se, $\dot{G}(t)=0$.

Pertanto, allo scopo di ntilizzare i Teoremi del n. 2, converrà riportarsi (quando ciò non si risolva in un artificio puramente formale) al caso in cui la condizione (3) risulti soddisfatta, scrivendo ad es., ove occorra, il sistema (1) nella forma

$$
\dot{x}=x+\{f(t, x)+[A(t)-I] x\},
$$

e riferendo la (10) e la (11) al vettore

$$
f_{1}(t, x)=f(t, x)+[A(t)-I] x .
$$

Un'applicazione di ciò sarà fatta, per esmpio, nel successivo n. $11\left(^{4}\right)$.

\section{4. - Torniamo alle ipotesi del n. 2.}

Dalle considerazioni ivi svolte appare chiaro che, per applicare ad es. il Teorema 1 al problema dell' esistenza di soluzioni periodiche di periodo $\omega$ del sistema (1), occorrerà non solo provare che sono non vuoti gli insiemi $U$ e $V$ costituiti dai vettori $u(t)$ soddisfacenti rispettivamente la (8) e la (7), ma che non è vuota altresi la loro intersezione.

Lo stesso dicasi, nel caso del Teorema 1', per gli insiemi $U^{\prime}$ e $V^{\prime}$ costituiti dai vettori di $Q$ per cui è soddisfatta rispettivamente la (10) o la (11).

Ora non è difficile riconoscere come tale procedimento presenti in generale notevoli difficoltà.

Ciò dipende principalmente dal fatto che gli insiemi $U, V\left[U^{\prime}, \nabla^{\prime}\right]$ non hanno sempre una struttura ben definita (ad es. di spazio lineare), e che il secondo membro della (8) $[(10)]$ non trasforma in genere l'insieme $V\left[V^{\prime}\right]$ in sè. E ciò esclude di regola la possibilità di far ricorso ai classici procedimenti dell' analisi funzionale.

(4) Confronta anche R. ReIssio, [7]. 
Si hanno tuttavia due casi «limite» in cui l'applicazione dei Teoremi 1 e $1^{\prime}$ si presenta in forma particolarmente agevole.

a) Il primo di questi è il caso in cui il vettore $f(t, x)$ non dipende da $x$, ed il sistema (1) si riduce alla forma

$$
\dot{x}=A(t) x+a(t)
$$

con $\alpha(t)$ vettore continuo per $t>T$.

In tale ipotesi la (8) assume la forma

$$
u(t)=Y(t)[Y(t)-Y(t+\omega)]^{-1} Y(t+\omega) \int_{t}^{t+\omega} Y^{-1}(\tau) a(\tau) d \tau
$$

ed è quindi univocamente soddisfatta.

Ne segue che gli insiemi $U$ e $V$ hanno intersezione non vuota se, e soltanto se, il vettore (15) verifica la condizione

$$
[A(t)-A(t+\omega)] u(t)+a(t)-a(t+\omega)=0 .
$$

In conclusione, dal Teorema 1 si ha il

Teorema 2. - Se per il sistema omogeneo (2) è soddisfatta la (3), condi. zione necessaria e sufficiente per l'esistenza di soluzioni periodiche di periodo w del sistema (14) è che si abbia

$$
\begin{gathered}
{[A(t)-A(t+\omega)] Y(t)[Y(t)-Y(t+\omega)]^{-1} Y(t+\omega) \int_{i}^{t+\omega} Y^{-1}(\tau) a(\tau) d \tau+} \\
+a(t)-a(t+\omega)=0 ;
\end{gathered}
$$

una tale soluzione, se esiste, è unica ed è rappresentata dal vettore (15).

Si noti che, se nel sistema (14) la matrice $A(t)$ ed il vettore $a(t)$ si suppongono periodici di periodo $\omega$, la (16) əे certamente soddisfatta. Si ritrova cosi un ben noto risultato relativo all' esistenza di soluzioni armoniche per i sistemi periodici (14) in condizioni di non risonanza (cfr. [8], p. 467).

b) Il secondo caso «limite», al quale peraltro è dedicata la quasi totalità delle ricerche esistenti sull' argomento del presente lavoro, si presenta quando la matrice $A(t)$ e il vettore $f(t, x)$ sono periodici rispetto a $t$ con periodo $\omega$.

In queste ipotesi infatti la (11) risulta identicamente soddisfatta, e l'in. sieme $V^{\prime}$ coincide con l'intero spazio $\Omega$ dei vettori periodici di periodo $\omega$. 
Ne segue che gli insiemi $U^{\prime}$ e $V^{\prime}$ avranno intersezione non vuota se, e soltanto se, risulterà non vuoto l'insieme $U^{\prime} \subset \Omega$; e, per il Teorema 1', il problema dell'esistenza di soluzioni periodiche di periodo $\omega$ del sistema (1) è ricondotto a quello della esistenza di soluzioni della (10) nello spazio. $\mathbf{Q}$.

Va osservato che, per la periodicità di $A(t)$, la (10) può assumere una forma più semplice, e così pure la condizione (3) che (come è stato già notato) si riduce in tal caso all'ordinaria ipotesi di non risonanza.

Ma di cio tratteremo con maggiori dettagli nei numeri 6 e seguenti.

5. - Prima di passare all' esame dei sistemi (1) di tipo periodico, riteniamo non inutile segnalare qualche semplice osservarione relativa al caso in cai per $t>T$ si abbia

$$
A(t+\omega)=H A(t), \quad f(t+\omega, x)=K f(t, x),
$$

con $H$ e $K$ matrici costanti.

Tali condizioni, che possono pensarsi come una naturale generalizzazione del caso dei sistemi periodici, in cui $H=K=I$, si presentano ad es. qualora per $A(t)$ e $f(t, x)$ valga una jpotesi di «antiperiodicita » con periodo $\omega$ $(H=K=-I)$.

a) Sia $\operatorname{det}(I-H) \neq 0$.

Ogni vettore $x(t)$, soluzione periodica di periodo $\omega$ del sistema (1), verificherà per la (11) la condizione

$$
A(t) x(t)=(I-H)^{-1}(K-I) f[t, x(t)],
$$

e sarà quindi anche soluzione del sistema

$$
\dot{x}=(I-H)^{-1}(K-H) f(t, x)
$$

In particolare:

se $H=K$, le eventuali soluzioni periodiche di periodo $\omega$ di (1) non possono che essere costanti;

se $K=I$, per ciascana di tali soluzioni si ha $A(t) x(l)=0$. Quindi, ad es., se non è identicamente det $A(t)=0$, e se è garantita l'unicità delle soluzioni di (1) rispetto ai dati iniziali, l'unica soluzione di tale sistema perio. dica di periodo $\omega$ è la nulla, qualora, e solo quando, si abbia $f(t, 0)=0$.

b) Sia det $(I-K) \neq 0$.

In tal caso, se $x(t)$ è nna soluzione periodica di periodo o di (1), dalla 
(11) si ha

$$
f[t, x(t)]=(I-K)^{-1}(H-I) A(t) x(t),
$$

e quindi $x(t)$ è anche soluzione del sistema omogeneo

$$
\dot{x}=(I-K)^{-1}(H-K) A(t) x .
$$

In particolare:

se $H=K$, si ottiene lo stesso risultato che in $a$ );

se $H=I$, ogni soluzione periodica di periodo $\omega$ di (1) è soluzione del sistema (2). Quindi, ad es., se vale la (3), l' unica di tali soluzioni è la nulla, qualora, e solo quando, si abbia $f(t, 0)=0$.

Va infine notato come nei casi qui trattati non si richieda esplicitamente che sia soddisfatta la (3). Infatti, a prescindere dalla validità di tale ipotesi, si ricava facilmente la necessità della(11) per l'esistenza di soluzioni periodiche di periodo $\omega$ del sistema (1).

6. - In questo, e nei numeri seguenti, oi riferiremo al caso di sistemi (1) periodici con periodo $\omega$, supponendo che la matrice $A(t)$ ed il vettore $f(t, x)$ soddisfino, oltre quella di continuità, le ipotesi

$$
A(t+\omega)=A(t), \quad f(t+\omega, x)=f(t, x) .
$$

Poichè, com' ̀̀ noto, per la matrice fondamentale $Y(t)$ del sistema (2) in tal caso si ha: $Y(t+\omega)=Y(t) Y(\omega)$, la condizione (3) si traduce in quella di non risonanza

$$
\operatorname{det}[l-Y(\omega)] \neq 0
$$

e corrisponde all'assenza di soluzioni periodiche di periodo $\omega$ del sistema (2) diverse dalla nulla.

Inoltre la (10) assume la forma

$$
x(t)=Y(t)[I-Y(\omega)]^{-1} Y(\omega) \int_{t}^{t+\omega} Y^{-1}(\tau) f[\tau, x(\tau)] d \tau
$$

e poichè la (11), per le (17), risulta identicamente soddisfatta, dal Teorema $1^{\prime}$ si ottiene che il problema dell'esistenza di soluzioni del sistema (1) periodiche di periodo w è ricondotto a quello dell' esistenza di punti uniti per la tra- 
sformazione

$$
T[x(t)]=Y(t)[I-Y(\omega)]^{-1} Y(\omega) \int_{t}^{t+\omega} Y^{-1}(\tau) f[\tau, x(\tau)] d \tau
$$

nello spazio $\Omega$ dei vettori $x(t)$ periodici di periodo $\omega$.

Definiamo, come di consueto, la norma in $\Omega$ ponendo

$$
\left.|x|=\max _{t_{0} \leq t \leq t_{0}+\omega}\|x(t)\|, \mid\|x(t)\|=\left(\frac{n}{\Sigma}\left|x_{i}(t)\right|^{2}\right)^{1 / 2}\right]
$$

poniamo inoltre:

$$
\left\{\begin{array}{l}
A=\max _{t_{0} \leq t \leq t_{0}+\omega}\|A(t)\|, \quad\left[\|A(t)\|=\left(\Sigma a^{2}{ }_{i j}(t)\right)^{1 / 2}\right], \\
B=\max _{t_{0} \leq t \leq t_{0}+\omega}\left\|Y(t)[I-Y(\omega)]^{-1} Y(\omega)\right\| \\
C=\max _{t_{0} \leq t \leq t_{0}+2 \omega}\left\|Y^{-1}(t)\right\|, \quad M_{r} \geq \max _{\substack{t_{0} \leq t \leq t_{0}+\omega \\
0 \leq\|x\| \leq r}}\|f(t, x)\| .
\end{array}\right.
$$

Si osservi che, se $x \in \Omega$, avendosi

$$
\begin{aligned}
& Y(t+\omega)[I-Y(\omega)]^{-1} Y(\omega) \int_{t+\omega}^{t+2 \omega} Y^{-1}(\tau) f[\tau, x(\tau)] d \tau= \\
= & Y(t)[1-Y(\omega)]^{-1} Y(\omega) \int_{t+\omega}^{t+2 \omega} Y^{-1}(\tau-\omega) f[\tau, x(\tau)] d \tau= \\
= & Y(t)[I-Y(\omega)]^{-1} Y(\omega) \int_{t}^{t+\omega} Y^{-1}(s) f[s, x(s)] d s,
\end{aligned}
$$

anche $T(x) \in \mathbf{Q}$.

La (20) trasforma quindi lo spazio $\mathbf{Q}$ in sè.

Essa è poi manifestamente continua, per la supposta continuità del rettore $f(t, x)$.

Inoltre, fissato $r>0$, da $|x| \leq r$ si ottiene

$$
|T[x(t)]| \leq B C \omega M_{r}
$$

ed avendosi

$$
\frac{d T[x(t)]}{d t}=A(t) T[x(t)]+f[t, x(t)]
$$


si ha pure

$$
\left|\frac{a T[x(t)]}{d t}\right| \leq(A B C \omega+1) M_{r}
$$

La (20), per un noto teorema di ARzelà, trasforma allora ogni insieme limitato di $\Omega$ in un insieme relativamente compatto, e può concludersi che essa è una trasformazione completamente continua su $\Omega$.

Ció consente, nello studio dell'esistenza di punti uniti della (20), di ri. correre al seguente

Lemma: La trasformazione (20) presenta punti uniti, nello spazio dei vettori periodici di periodo $\omega$, se non è vuoto l'insieme $J$ dei numeri positivi $r$ tali che

$$
B C \omega M_{r} \leq r
$$

Infatti, se $r_{0} \in J$, indicando con $S_{0}$ la sfera di $\Omega$ costituita dai vettori di norma non superiore a $r_{0}$, da $x \in S_{0}$ si ha

$$
|T(x)| \leq\left(B C \omega M_{r_{0}}-r_{0}\right)+r_{0} \leq r_{0} .
$$

Ne segue che la trasformazione completamente continua (20) trasforma $S_{0}$ in sè, e per completare la dimostrazione del Lemma basta applicare un noto teorema di Schauder (cfr. [9]; ofr. anche [2], p. 61).

ha nel

Teorema 3. - Siano soddisfatte, per la matrice $A(t)$ e per il vettore $f(t, x)$, le ipotesi (17) e (18). Esiste allora per il parametro reale $\lambda$ un intorno dell'origine in corrispondenza del quale il sistema

$$
\dot{x}=A(t) x+\lambda f(t, x)
$$

presenta soluzioni periodiche di periodo $\omega$.

Basta infatti osservare che ogni numero $r>0$ può pensarsi apparte. nente all'insieme $J$ corrispondente ad un sistema (22) in cui

$$
|\lambda| \leq \frac{r}{B C \omega M_{r}}
$$

Tale teorema può essere messo in relazione con risultati classici della 
teoria della perturbazione (cfr. ad es. [5], p. 113) in cui, in ipotesi di maggiore regolarità per il vettore $f(t, x)$, e per $\lambda$ sufficientemente piccolo, si prova inoltre l'unicita delle soluzioni periodiche di periodo w della (22) e la loro dipendenza continua rispetto a $\lambda$.

Esso esprime in sostanza che la non risonanza della parte lineare di un sistema periodico (1) garantisce la persistenza di soluzioni periodiche nei confronti di «piccole» perturbazioni continue.

Va comunque esplicitamente sottolineato che, qualora si abbandoni la ipotesi della non risonanza, tale proprietà cessa in generale di sussistere.

Per vederlo basta rifarsi al classico esempio dell' equazione

$$
\dot{x}+x=\lambda \text { sent }
$$

che, per qualunque valore del parametro $\lambda \neq 0$, risulta priva di soluzioni periodiche di periodo $2 \pi$.

Un'ulteriore applicazione del Lemma precedente può essere fatta nel caso dei sistemi (1) in cui, oltre la (17) e la (18), risulti soddisfatta la condizione

$$
\|f(t, x)\| \leq a\|x\|^{\alpha}+b
$$

con $\alpha, a, b$ costanti reali non nega tive.

In realtà basterà limitarsi al caso $\alpha \neq 1$, poichè per $\alpha=1$ un risultato di BaRbãlat e HaLANay [1] garantisce l'esistenza di soluzioni periodiche di periodo $\omega$ del sistema (1) qualora si abbia

$$
a \leq \frac{1}{B C \omega} .
$$

Cominciamo con l'osservare che, se $0 \leq \alpha<1$, non è vuoto l'insieme dei numeri $r>0$ per oni risulta

$$
\begin{gathered}
B C \omega\left(a r^{\alpha}+b\right)-r= \\
=r\left(\frac{B C \omega}{r^{1-\alpha}}-1\right)+B C \omega b \leq 0
\end{gathered}
$$

e non è quindi vuoto in nessun caso l'insieme $J$ che figura nell' enuneiato del Lemma.

Sia dunque $\alpha>1$. Potrà applicarsi il Lemma quando si provi che la funzione

$$
\Gamma(\boldsymbol{r})=B C \omega r^{x}-r+B C \omega b
$$


assume valore non positivo in qualche punto della semiretta $r>0$. Si vede facilmente che il minimo per la (25) si ottiene quando

e perchè si abbia

$$
r=r_{\alpha}=(\alpha B C \omega a)^{-\frac{1}{x-1}}
$$

occorre che sia

$$
\begin{aligned}
& \Gamma\left(r_{\alpha}\right)=B O \omega \alpha(\alpha B C \omega a)^{-\frac{z}{\alpha-1}}- \\
& -(\alpha B C \omega a)^{-\frac{1}{\alpha-1}}+B C \omega b \leq 0
\end{aligned}
$$

$$
a \cdot b^{\alpha-1} \leq(\alpha-1)^{\alpha-1} \frac{1}{(\alpha B C \omega)^{\alpha}}
$$

Coneludendo si ha il

Teorema 4. - Se sono soddisfatte le ipotesi (17), (18) e (23), il sistema (1) presenta soluzioni periodiche di periodo w per $0 \leq \alpha<1$, e, qualora valga la (26), per $\alpha>1$.

Si osservi che, avendosi $\lim _{x \rightarrow 1+}(\alpha-1)^{x-1}=1$, la condizione (24) di BaRBäLat e Halanay si ottiene dalla (26) facendo tendere $\alpha \rightarrow 1$ per valori $>1$.

Inoltre, se $b=0$, la (26) ¿े certamente soddisfatta; e ne segue che, in quest' ultima ipotesi, $\alpha \geq 0, \alpha \neq 1$ è sufficiente a garantire l'esistenza di soluzioni periodiche di periodo w del sistema (1).

8. - L'ultima parte della ricerca è dedicata al problema dell'esistenza di soluzioni periodiche di periodo $\omega$ per le equazioni del $3^{\circ}$ ordine del tipo (di Atzerman).

$$
\dddot{u}+a \ddot{u}+\dot{b u}+c u=p(t)+q(\ddot{u})+r(\dot{u})+s(u),
$$

ove $a, b, c$ sono costanti reali, $p(t), q(w), r(v), s(u)$ sono funzioni continue dei loro argomenti, ovunque definite, ed inoltre

$$
p(t+\omega)=p(t)
$$

La (27) rientra nella classe dei sistemi (1) ove si ponga

$$
\left\{\begin{array}{c}
u=x_{1}, \quad \dot{u}=x_{2}, \quad \ddot{u}=x_{3} \\
A(t)=\left(\begin{array}{rrr}
0 & 1 & 0 \\
0 & 0 & 1 \\
-c & -b & -a
\end{array}\right), f(t, x)=\left(\begin{array}{c}
0 \\
0 \\
p(t)+q\left(x_{3}\right)+r\left(x_{2}\right)+s\left(x_{1}\right)
\end{array}\right) ;
\end{array}\right.
$$


supporremo inoltre che le costanti $a, b, c$ siano tali da garantire la condizione di non risonanza $(18$;

Nello studio dell'esistenza di punti uniti per la corrispondente trasformazione (20) faremo ricorso al metodo di LERAX-SchadDER (ofr. [4]; ofr. anche [6], p. 152).

A tale scopo consideriamo la trasformazione

$$
T_{\lambda}(x)=\lambda Y(t)[I-Y(\omega)]^{-1} Y(\omega) \int_{t}^{t+\omega} Y^{-1}(\tau) f[\tau, x(\tau)] d \tau
$$

nella quảe $Y(t)$ e $f(t, x)$ si intendono riferiti al caso delle (29), e $\lambda$ è un parametro reale che supporremo variabile nell'intervallo $[0,1]$.

Per quanto è stato osservato al n. 6, le soluzioni della (30), nello spazio $\Omega$ dei vettori periodici di periodo $\omega$, sono tutte e sole le soluzioni periodiche, di periodo $\omega$, dell'equazione

$$
\ddot{u}+a \ddot{u}+b \dot{u}+c u=\lambda[p(t)+q(\ddot{u})+r(\dot{u})+s(u)] .
$$

Inoltre la $(30)$ è una trasformazione completamente continua sn $\Omega$, dipendente con continuita dal parametro $\lambda$, che per $\lambda=0$ trasforma ogni elemento di $\Omega$ nel suo elemento neutro, e per $\lambda=1$ coincide con la (20).

Pertanto, quando si provi che, per $0 \leq \lambda \leq 1$, le eventuali soluzioni di (30) in $\Omega$, cioè le eventuali soluzioni periodiche di periodo w della (31), sono ugualmente limitate, si potrà concludere che la (20) presenta soluzioni nello spazio $\Omega$ e quindi l'equazione (27) è dotata di soluzioni periodiche di periodo $\omega$.

9. - In questo, e noi numeri seguenti, applicheremo il metodo indicato nel precedente paragrafo per ottenere alcuni criteri di esistenza di soluzioni periodiche nel caso di equazioni del tipo (27).

Cominciamo col dimostrare il

Thorema 5. - Se sono soddisfatte le condizioni

$$
\begin{gathered}
b<0, \quad c \neq 0, \\
|s(u)| \leq m<+\infty,
\end{gathered}
$$

e se inoltre si ha

$$
r^{\prime}(v) \geq 0, \quad \text { ovvero } \quad\left(34^{\prime}\right) \quad|r(v)| \leq m<+\infty
$$

l'equazione (27) presenta almeno una soluzione periodica di periodo $\omega$. 
Si osservi anzitutto che le (32) garantiscono la validità della (18) sia per la (27) che per la (31); infatti l'equazione caratteristica della rispettiva equazione omogenea associata risulta priva di radici con parte reale nulla.

Ciò posto, fissato $\lambda(0 \leq \lambda \leq 1)$, indichiamo con $u=z(t)$ una (eventuale) soluzione periodica di periodo $\omega$ della equazione (31).

Moltiplicando per $z(t)$ ed integrando tra 0 e $\omega$, dalla (31) si ha

$$
\int_{0}^{\omega} \ddot{z}^{2}(t) d t-b \int_{0}^{\omega} \ddot{z}^{2}(t) d t=\lambda \int_{0}^{\omega} \ddot{z}(t)\{p(t)+r[\dot{z}(t)]+s[z(t)]\} d t
$$

ovvero

$$
\int_{0}^{\omega} \ddot{z}^{2}(t) d t-b \int_{0}^{\omega} \ddot{z}^{z}(t) d t=\lambda \int_{0}^{\omega} \ddot{z}(t)\{p(t)+s[z(t)]\} d t-\lambda \int_{0}^{\omega} \ddot{z}^{2}(t) r^{\prime}[\dot{z}(t)] d t .
$$

In entrambi i casi, dalle (32), (33), e per la prima, ovvero per la seconda, delle (34), ponendo $p=\max |p(t)|$ ed applicando una nota disuguaglianza, si ottiene

$$
\begin{gathered}
\int_{0}^{\omega} \ddot{z}^{2}(t) d t+|b| \int_{0}^{\omega} \ddot{z^{2}}(t) d t \leq \int_{0}^{\omega}\left|\ddot{z}^{2}(t)\right|(p+2 m) d t \leq \\
\leq\left(\int_{0}^{\omega} \ddot{z}^{2}(t) d t\right)^{1 / 2}\left(\int_{0}^{\omega}(p+2 m)^{2} d t\right)^{1 / 2},
\end{gathered}
$$

e da questa

$$
\int_{0}^{\omega} \ddot{z}^{2}(t) d t<L, \quad \int_{0}^{\omega} \ddot{z^{2}}(t) d t<L
$$

ove

$$
L>\max \left[\omega(p+2 m)^{2}, \frac{\omega}{|b|}(p+2 m)^{2}\right] .
$$

Osserviamo che le (35) consentono di stabilire una limitazione per $|\ddot{z}(t)|$.

Infatti, posto $M=\max (1,2 L, L / \omega)$, ragioniamo per assurdo supponendo che in un punto $t_{1} \in[0, \omega]$ si abbia $\ddot{z}^{2}\left(t_{1}\right)>2 M$. Non potrà essere $\ddot{z}^{2}(t)>M$ per $t \in[0, \omega]$, poichè in tal caso si avrebbe

$$
\int_{0}^{\omega} \ddot{z^{2}}(t) d t \geq \int_{0}^{\omega}|\ddot{z}(t)| d t>M \omega \geq L
$$

in contrasto con la seconda delle (35). 
Esisterà quindi almeno un punto $t_{2} \in[0, \omega]$ in cui $\ddot{\Xi}^{2}\left(t_{2}\right)=M$; ma poichè sarebbe

$$
\begin{gathered}
M<\left|\ddot{z}^{2}\left(t_{2}\right)-\ddot{z}^{2}\left(t_{1}\right)\right| \leq 2 \int_{t_{1}}^{t_{2}}|\ddot{z}(t)||\ddot{z}(t)| d t \leq \\
\left(\int_{0}^{\omega} \ddot{z^{2}}(t) d t\right)^{1 / 2}\left(\int_{0}^{\omega} \ddot{z}^{2}(t) d t\right)^{1 / 2}<2 L \leq M,
\end{gathered}
$$

si cade in assurdo.

Resta così provata la limitazione

$$
\ddot{z}^{2}(t)<2 M
$$

e sia

$$
|q(w)| \leq m_{2} \quad \text { per }|w|<\sqrt{2 M}
$$

Ancora dalla (31), moltiplicando per $\ddot{z}(t)$ ed integrando tra 0 e $\omega$, otteniamo

$$
\alpha \int_{0}^{\omega} \ddot{z^{2}}(t) d t-c \int_{0}^{\omega} \dot{z}^{2}(t) d t=\lambda \int_{0}^{\omega} \ddot{z}(t)\{p(t)+q[\ddot{z}(t)]+s[z(t)]\} d t,
$$

da cui

$$
|c| \int_{0}^{\omega} \dot{z}^{2}(t) d t<2 M|a| \omega+2 M\left(p+m+m_{2}\right) \omega .
$$

Ne segue una limitazione per l'integrale di $\dot{z}^{2}(t)$ che, per semplicità di notazione, scriveremo ancora

$$
\int_{0}^{\omega} \dot{z}^{2}(t) d t<L
$$

e da questa, con procedimento analogo a quello usato nel caso di $\ddot{z}(t)$, si ottiene

$$
\dot{z}^{2}(t)<2 M
$$

Sia infine

$$
|r(v)| \leq m_{1} \quad \text { per }|v|<\sqrt{2 M}
$$


Moltiplicando per $\approx(l)$ ed integrando tra 0 e $(0$, sempre dalla (31) si ha

$$
-a \int_{0}^{t} \dot{z}^{2}(t) d t+c \int_{0}^{\omega} z^{2}(t) d t=\lambda \int_{0}^{\omega} z(t)\{p(t)+q[\ddot{z}(t)]+r[\dot{z}(t)]+s[z(t)]\} d t,
$$

da cui

$$
|c| \int_{0}^{\omega} z^{2}(t) d t \leq \int_{0}^{\omega}|z(t)|\left(p+m+m_{1}+m_{2}\right) d t+2 M|a| \omega .
$$

Ne segue, anche in tal caso, una limitazione per l'integrale di $z^{2}(t)$, che scriveremo

$$
\int_{0}^{\omega} z^{2}(t) d t<L
$$

e quindi

$$
z^{2}(t)<2 M
$$

In conclusione, per $\lambda \geq 0, \lambda \leq 1$, se $u=z(t)$ è una soluzione periodica di periodo $\omega$ della corrispondente equazione (31), si ottengono le limitazioni (36), (37), (38).

Si ha da qui che le eventuali soluzioni in $\Omega$ dell'equazione

$$
T_{\lambda}(x)-x=0
$$

risultano egualmente limitate per $0 \leq \lambda \leq 1$, e dalle considerazioni del paragrafo precedente ne segue l'esistenza in $\Omega$ di almeno un punto unito per la trasformazione $(20)$.

Ciò completa la dimostrazione del Teorema 5.

10. - Sempre per l'equazione (27), un risultato analogo a quello contenuto nel precedente Teorema si ha col

Teorema 6. - Se sono soddisfatte le condizioni

$$
\begin{aligned}
& a c<0 \text {, } \\
& c w q(w) \geq 0 \\
& \operatorname{aus}(u) \geq 0, \quad a s^{\prime}(u) \geq 0, \quad\left|s^{\prime \prime}(\boldsymbol{u})\right| \leq h<+\infty,
\end{aligned}
$$

l'equazione (27) presenta almeno una soluzione periodica di periodo $\omega$. 
Poichè la dimostrazione procede in modo simile a quella del Teorema 5 , ei limitiamo ad indicarne le linee essenziali senza entrare in eceessivi dettagli.

Anche in tal caso cominciamo con l'osservare che la (39) garantisce la validità della (18), sia per la (27) che per la (31); infatti l'equazione caratteristica della corrispondente equarione omogenea associata risulta priva di radici con parte reale nulla.

Indichiamo con $u=z(t)$ una generica soluzione periodica di periodo $\omega$ della (31).

Moltiplicando per $\ddot{z}(t)$ ed integrando sull'intervallo $[0, \omega]$, dalla (31) si ha

$$
\begin{gathered}
a \int_{0}^{\omega} \ddot{z^{2}}(t) d t-c \int_{0}^{\omega} \dot{z}^{2}(t) d t=\lambda \int_{0}^{\omega} p(t) \ddot{z}(t) d t+ \\
\quad+\lambda \int_{0}^{\omega} \ddot{z}(t) q[\ddot{z}(t)] d t-\lambda \int_{0}^{\omega} \dot{z}^{2}(t) s^{\prime}[\boldsymbol{z}(t)] d t,
\end{gathered}
$$

e quindi, per la (39), (40) e per la seconda delle (41)

$$
\begin{gathered}
|\alpha| \int_{0}^{\omega} \ddot{z^{2}}(t) d t+|c| \int_{0}^{\omega} z^{2}(t) d t \leq \int_{0}^{\omega}|p(t)||\ddot{z}(t)| d t \leq \\
\leq\left(\int_{0}^{\omega} p^{2}(t) d t\right)^{1 / 2}\left(\int_{0}^{\omega} \ddot{z}^{2}(t) d t\right)^{1 / 2} .
\end{gathered}
$$

Ne segue

$$
\int_{0}^{\omega} z^{2}(t) d t<L, \quad \int_{0}^{\omega} z^{2}(t) d t<L,
$$

e per $z(t)$ la limitazione

$$
|\dot{z}(t)|<k \text {. }
$$

Sia $\left|r^{\prime}(v)\right|<h_{1}$ per $|v|<k$.

Ancora dalla (31), moltiplicando per $\stackrel{*}{z}(t)$ ed integrando sull'intervallo $[0, \omega]$, si ottiene

$$
\begin{aligned}
& \int_{0}^{\omega} \ddot{z}^{2}(t) d t-b \int_{0}^{\omega} \ddot{z^{2}}(t) d t=\lambda \int_{0}^{\omega} p(t) \cdots(t) d t- \\
& -\lambda \int_{0}^{\omega} \ddot{z}(t) r^{\prime}[\dot{z}(t)] d t+\frac{\lambda}{2} \int_{0}^{\omega} \dot{z}^{3}(t) s^{\prime \prime}[z(t)] d t,
\end{aligned}
$$


da cui, per la terza delle (41) e le precedenti limitazioni :

$$
\int_{0}^{0} \dddot{z}^{2}(t) d t<\int_{0}^{0} p|\cdots(t)| d t+\left(|b|+h_{1}\right) L+{ }_{2}^{1} h k^{3} \omega .
$$

Ciò consente di stabilire anche per $\ddot{z}(t)$ una limitazione simile alla (42), e sia

$$
|q(w)|<h_{2} \text { per }|w|<k \text {. }
$$

Infine, moltiplicando la (31) per $z(t)$ ed integrando tra 0 e $w$, per la prima delle (41) si ha

$$
|c| \int_{0}^{\omega} z^{2}(t) d t \leq \int_{0}^{\omega}\left(p+h_{1}+h_{2}\right)|z(t)| d t+|a| L
$$

e ne segue una limitazione per $|z(t)|$.

In conclusione, le eventuali soluzioni periodiche di periodo $\omega$ risultano egualmente limitate per $0 \leq \lambda \leq 1$, e per completare la dimostrazione è suf* ficiente applicare le conclusioni del n. 8.

Si osservi ohe la prima delle (41) può essere sostituita dalla condizione

$$
|s(\boldsymbol{u})| \leq h<+\infty
$$

Infatti la (43) diventa

$$
|c| \int_{0}^{\omega} z^{2}(t) d t \leq \int_{0}^{\omega}\left(p+|a| h+h_{1}+h_{2}\right)|z(t)| d t,
$$

il ohe non altera il procedimento.

11. - Dai Teoremi 5 e 6 si deduce che le sole ipotesi (32) e (39) sono sufficienti a garantire l'esistenza di soluzioni periodiche di periodo $\omega$ per le equazioni

$$
\dddot{u}+q(\ddot{u})+b \dot{u}+c u=p(t)
$$

e

$$
\ddot{u}+a \ddot{u}+r(\dot{u})+c u=p(t) .
$$

Ciò migliora noterolmente, per la parte concernente l'esistenza, due nostri precedenti criteri (cfr. [10], pp. 77 e 82), e conferma la congettura (cfr. [10], 
p. 72) che simili risultati potessero stabilirsi in ipotesi di sola non risonanza, quali sono appunto le (32) e la (39).

Meno diretta appare invece l'applicazione del procedimento dimostrativo indicato al n. 8 al caso dell'equazione

$$
\ddot{u}+a \ddot{u}+b \dot{u}+s(u)=p(t)
$$

in cui, non presentandosi la parte lineare in condizioni di non risonanza, viene a mancare la possibilità di ricorrere ad una trasformazione come la (20).

Ad es., in alcuni criteri riguardanti l'equazione (44) (cfr. [3], [5]), la definizione di una trasformazione completamente continua in $E^{n}$, che consenta di applicare il metodo di LERAY-SOHAUDER, comporta un notevole appesan. timento delle ipotesi, fra cui quello della limitatezza di tatte le soluzioni della (44).

Non mancano tuttavia casi in cui, seguendo l'osservazione fatta alla fine del n. 3, è possibile applicare il procedimento usato per la dimostrazione dei due ultimi Teoremi.

Tra tali casi, a titolo di esempio, segnaliamo il criterio contenuto nel

Thormana 7. - Sia $p(t)$ continua e periodica di periodo w; sia inoltre $s(0)=0$, ed esista una costante c tale che

$$
a c<0, \quad \frac{s^{\prime}(u)}{c} \geq 1
$$

In tali condizioni l'equazione (44) presenta almeno una solusione periodica di periodo $\omega$.

Scriviamo infatti la (44) nella forma

$$
\dddot{u}+a \ddot{u}+b \dot{u}+c u=p(t)-[s(u)-c u]
$$

e, per $0 \leq \lambda \leq 1$, consideriamo l'equazione

$$
\dddot{u}+a \ddot{u}+b \dot{u}+c u=\lambda\{p(t)-[s(u)-c u]\} \text {. }
$$

La prima delle (45) garantisce, come si è già osservato, la non risonanza della parte lineare associata al primo membro della (47). Inoltre, posto $F(u)=s(u)-c u$, dalla seconda delle (45) si ha $c F^{\prime}(u) \geq 0$, e quindi

$$
c u[s(u)-c u] \geq 0
$$

Indichi $u=z(t)$ una generica soluzione periodica di periodo $\omega$ della $(47)$. 
Moltiplicando per $z(t)$ ed integrando sullintervallo $[0, \omega]$, dalla (47) si ottiene

$$
\begin{gathered}
-a \int_{0}^{\omega} z^{2}(t) d t+c \int_{0}^{\omega} z^{2}(t) d t=\lambda \int_{0}^{t} z(t) p(t) d t- \\
-\lambda \int_{0}^{\omega} z(t)\{s[z(t)]-c z(t)\} d t
\end{gathered}
$$

e dalla (48) :

$$
|a| \int_{0}^{\omega} z^{2}(t) d t+|0| \int_{0}^{\omega} z^{2}(t) d t \leq \int_{0}^{\omega}|z(t)||p(t)| d t .
$$

Ne segue una limitazione per $z(t) \mid$, e poichè la parte non lineare della (47) dipende solo da $z(t)$, può concludersi che, per $0 \leq \lambda \leq 1$, le eventuali soluzioni in $\Omega$ della corrispondente equazione

$$
T_{\lambda}(x)-x=0
$$

sono ugnalmente limitate.

\section{BIBLIOGRAFIA}

[1] I. BaRbarät - A. HALANAY, Solutions périodiques des systèmes d'équations différentielles non linéaires, Revue de Mathématiques pures et appliquées, 3 (1958), 395-412.

[2] R. Cont, Equazioni differenziali ordinarie quasilineari con condizioni lineari, Ann. di Mat. pura ed appl, Serie IV, 57 (1962), 49.61.

[0] J.0.C. EzErLo, On the existence of periodic solutions of a certain bind-order differential equation, Proc. Cambridge Philos. Soc., 56 (1960), 381.389.

[4] J. Leray - J. Schauder, Topologie et équations fonctionnelles, Aun. Fo. Norm. Sup., S. III, 51 (1984), $400-78$.

[5] J.G. MALKIN, Alcuni problemi della teoria delle oscillazioni non lineari, Mosea (1056) (in russo).

[6] C. Mranda, Problemi di esistenza in analisi funzionale, Quaderni mat. S N.S. Pisa, n. $3,(1948.49)$.

[7] R. REISsig, Ein funhtionalanalytischer Existenzbeneis für periodische Lösungen, Monatber. d. Deut. Ak. d. Wiss. zu Berlin, 6 (1961), 407.413.

[8] G. SANsone - R. Contr, Non linear differential equalions, Pergamon Press (1964).

[9] J. Schauder, Der Fixpunktsate in Funktonalramen, Stndia Math., 2 (1980), 171-180.

[10] G. VILLARI, Criteri di esistenza di soluzioni periodiche per particolavi classi di equazioni differenziali deb ters'ordine non lineari Le Matematiche, 19 (1964), 70-86. 